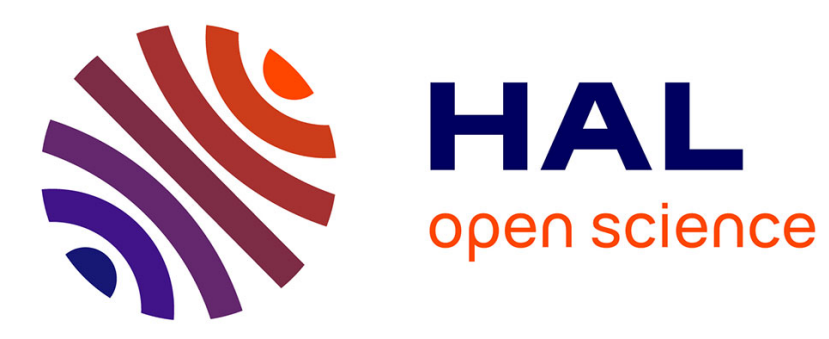

\title{
Cancellation time for correlated random walkers
}

Fernando Arias de Saavedra, Malvin H Kalos, Francesco Pederiva

\section{To cite this version:}

Fernando Arias de Saavedra, Malvin H Kalos, Francesco Pederiva. Cancellation time for correlated random walkers. Molecular Physics, 2011, pp.1. 10.1080/00268976.2011.604647 . hal-00722800

\section{HAL Id: hal-00722800 \\ https://hal.science/hal-00722800}

Submitted on 4 Aug 2012

HAL is a multi-disciplinary open access archive for the deposit and dissemination of scientific research documents, whether they are published or not. The documents may come from teaching and research institutions in France or abroad, or from public or private research centers.
L'archive ouverte pluridisciplinaire HAL, est destinée au dépôt et à la diffusion de documents scientifiques de niveau recherche, publiés ou non, émanant des établissements d'enseignement et de recherche français ou étrangers, des laboratoires publics ou privés. 


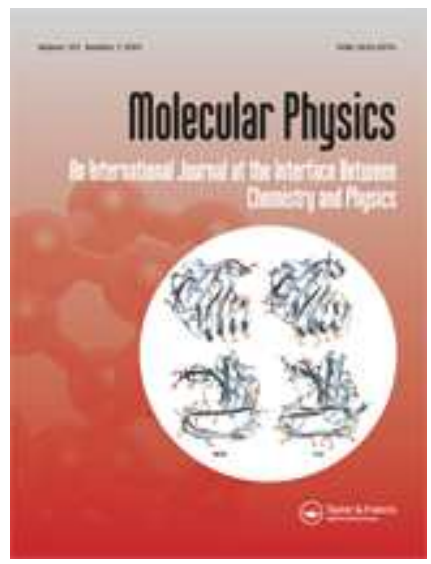

\section{Cancellation time for correlated random walkers}

\begin{tabular}{|r|l|}
\hline Journal: & Molecular Physics \\
\hline Manuscript ID: & TMPH-2011-0206 \\
\hline Manuscript Type: & Special Issue in honour of Luciano Reatto \\
\hline Author: & 26-Jun-2011 \\
\hline Complete List of Authors: & $\begin{array}{l}\text { Arias de Saavedra, Fernando; Universidad de Granada, Fisica } \\
\text { Atomica, Molecular y Nuclear } \\
\text { Kalos, Malvin; Lawrence Livermore National Lab. } \\
\text { Pederiva, Francesco; Universita di Trento, Fisica }\end{array}$ \\
\hline Keywords: & $\begin{array}{l}\text { Quantum Monte Carlo, Fermion Monte Carlo, Diffusion Monte Carlo, } \\
\text { correlated walkers, harmonic oscillator }\end{array}$ \\
\hline & \\
\hline $\begin{array}{l}\text { Note: The following files were submitted by the author for peer review, but cannot be converted } \\
\text { to PDF. You must view these files (e.g. movies) online. }\end{array}$ \\
\hline paper.tex
\end{tabular}

\section{SCHOLARONE $^{\text {m }}$ Manuscripts}


Molecular Physics

Vol. 00, No. 00, Month 200x, 1-12

\title{
RESEARCH ARTICLE
}

\section{Cancellation time for correlated random walkers}

\author{
F. Arias de Saavedra ${ }^{\dagger}$, Malvin H Kalos, \\ and Francesco Pederiva $a^{\ddagger}$ \\ Lawrence Livermore National Laboratory \\ ${ }^{\dagger}$ Universidad de Granada, España \\ ¥Università di Trento, Italia \\ (Received 00 Month 200x; final version received 00 Month 200x)
}

\begin{abstract}
We have studied the dynamics of the correlated diffusion of pairs of random walkers of opposite signs. The use of populations of such pairs has been proposed for the Monte Carlo treatment of many-fermion systems, where the possibility of their cancellation might prevent the characteristic decay of signal-to-noise ratio. For four model systems- free fermions, the harmonic oscillator, an N-body system of attractive and repulsive harmonic forces, and an extensive system interacting by Pöschl-Teller potentials- we have explored analytically and by computation the behavior of the time to cancellation as a function of initial conditions and, equally important, as a function of system size. We find that for these systems the computational efficiency does not decay either with large imaginary time or with large $\mathrm{N}$.
\end{abstract}

Keywords: Quantum Monte Carlo, Fermion Monte Carlo, Diffusion Monte Carlo, correlated walkers, harmonic oscillator

\section{Introduction}

Quantum Monte Carlo (QMC) methods have become an important tool in the study of many-body systems. They permit accurate solutions of systems of bosons. No comparable methodology exists for fermionic systems, but with many theoretical and practical improvements, the fixed-node approximation has proved to be a powerful tool.

The impediments to a reliable many-fermion Monte Carlo method that does not require the specification of a nodal surface can be enumerated in several ways, but the following stands out: because the fermionic state has a larger eigenvalue than the bosonic, the projection of the walker distribution using an antisymmetric trial function decays exponentially fast in imaginary time, and the rate of decay grows with the particle number $N$ - linearly for extensive systems. Arnow et al. [1] proposed using walkers of opposite signs and canceling close pairs using Green's functions. This scales badly in particle number since near neighbors become exponentially rare in the many-dimensional configuration space used, and because accurate Green's functions can easily be constructed only over small Euclidean distances (or equivalently, over short intervals of imaginary time.)

Kalos and Pederiva have been studying for some time a class of methods intended to provide exact results for many-fermion systems that does not depend on the specification of a nodal surface $[2,3]$. A key element of those methods is a population of pairs of random walkers of opposite algebraic signs together with correlation of the diffusion steps of the random walks of the members of a pair in such a way that they can meet with high probability.

This paper is devoted to a critical analytic and experimental study of the corre- 
lated random walks to determine whether they are effective and especially to study the dependence on particle number-i.e., on dimensionality- of the dynamics of cancellation.

The current version of Fermion Monte Carlo (FMC) may be outlined as follows.

If the dimension of the physical space is $d$ then the walkers that generate the Monte Carlo solution of the Schrödinger equation move in a $d N$ dimensional space. We study the behavior of pairs of such walkers that carry plus and minus signs. Each is moved according to the dynamics of simple diffusion Monte Carlo using a trial function for the symmetric ground state as an importance function. The diffusion moves are correlated for the two walkers, and when close, they can cancel each other. Then a "second stage importance function," which depends on the coordinates of both walkers, is applied and the pair density is altered according to the ratio of this function before and after the time step.

Because the branching weight using the symmetric importance function may not be the same for both walkers, a special random process is invoked: half the difference between the two weights is given to the walker with the larger weight, which is then converted to a pair by applying an odd permutation. This creates no bias, since the permuted walker is given the opposite sign and has the same future expectation as its partner. This process is called "repairing".

Cancellation of the pairs is essential to avoid the exponential growth of the symmetric part of the walker density at the expense of the antisymmetric part. Of course, the a priori chance of finding a pair close enough to cancel in $d N$ dimensions falls exponentially fast with $N$. The correlation of the walkers has the effect of bringing them together so that cancellation becomes likely.

But the fermion-boson energy gap increases with $N$, growing linearly with $N$ for extensive systems, like ${ }^{3} \mathrm{He}$ or the homogeneous electron gas. The question arises whether the correlation process brings the walkers together fast enough for efficient cancellation in large systems.

This question has motivated a series of experiments to study the behavior of the cancellation time, i.e., the time necessary for a pair of walkers to get close and cancel. To avoid additional sources of uncertainty, we have made the experiments with three different systems with a known imaginary-time Green's function for any number of particles. These systems are the free gas, the harmonic oscillator, and an atom-like system in which an attractive central harmonic force competes with a weaker repulsive harmonic force between every pair of particles. This latter is a simple solvable analog of an atom, which we call the "harmonium atom."

\section{Cancellation time}

Let us denote by $G(X, Y ; \tau)$ the Green's function for vectors $X$ and $Y$ with $d N$ components describing a move from $Y$ to $X$ for a system of $N$ particles which move in a $d$-dimensional space; $\tau$ is the imaginary time. Let us consider a pair of walkers, denoted by $\left(X_{0}^{+}, X_{0}^{-}\right)$, comprising a positive walker, $X_{0}^{+}$, and a negative one, $X_{0}^{-}$. The probability of cancellation of walkers of opposite sign is described by the subtraction of Green's functions in the following way: the density of positive and negative walkers in the move of a pair from $\left(X_{0}^{+}, X_{0}^{-}\right)$to $\left(X^{+}, X^{-}\right)$will be:

$$
\begin{aligned}
& \rho^{+}\left(X ; X_{0}^{+}, X_{0}^{-}, \tau\right)=\max \left[0, G\left(X, X_{0}^{+} ; \tau\right)-G\left(X, X_{0}^{-} ; \tau\right)\right] \\
& \rho^{-}\left(X ; X_{0}^{+}, X_{0}^{-}, \tau\right)=\max \left[0, G\left(X, X_{0}^{-} ; \tau\right)-G\left(X, X_{0}^{+} ; \tau\right)\right]
\end{aligned}
$$

respectively. The move of the pair in FMC is the following: 
(1) We move $X_{0}^{+}$to $X^{+}$according to $G\left(X^{+}, X_{0}^{+} ; \tau\right)$.

(2) We accept $X^{+}$with probability

$$
\frac{\rho^{+}\left(X^{+} ; X_{0}^{+}, X_{0}^{-}, \tau\right)}{G\left(X^{+}, X_{0}^{+} ; \tau\right)}
$$

If the move is not accepted, the pair is canceled; counting the number of steps taken by the pair from birth to death gives its cancellation time.

(3) If accepted, we move $X_{0}^{-}$to $X^{-}$using

$$
X^{-}=X_{0}^{-}+X^{+}-X_{0}^{+}-2 \frac{\left(X^{+}-X_{0}^{+}\right) \cdot\left(X_{0}^{+}-X_{0}^{-}\right)}{\left(X_{0}^{+}-X_{0}^{-}\right)^{2}}\left(X_{0}^{+}-X_{0}^{-}\right) .
$$

This correlated move implies that $X^{-}$has the same probability of acceptance as $X^{+}, \mathrm{Eq}(3) \cdot\left(X^{+}, X^{-}\right)$is the new position of the pair; we go back to the step 1 if the moves are accepted.

It is obvious that, apart from number of particles, $N$, and the dimension of the space they move, $d$, the cancellation time will also depend on the initial distribution of the two walkers in the pair.

\section{Free gas}

In the case of the free gas, the exact Green function is:

$$
G(X, Y ; \tau)=\frac{1}{(2 \pi \tau)^{N d / 2}} \exp \left[-\frac{(X-Y)^{2}}{2 \tau}\right]
$$

The symmetric ground state wave function is constant.

We have to consider the infinite images of the vectors created by periodic boundary conditions. Since we know the behavior of this system as a function of density, we will take $\rho=1$. Then the $N$ particles are in a hypercube with side, $L$, with $L=N^{1 / d}$. In a practical calculation if $\tau$ is small enough compared to $L^{2}$, we can pair each $X$ with its single nearest image.

We begin by studying the behavior of cancellation time with the number of particles. As a first case, we will choose the positions of the walkers randomly and uniformly in the box of volume, $N$. We show the results in Figure 1 for $d=1,2,3$ as a function of the number of particles and compare to a fit in the form

$$
t_{c}=C N^{1+2 / d} .
$$

The values in Figure 1 correspond to $C=0.0412,0.0806,0.26$ for $d=1,2,3$, respectively. This behavior can be easily explained since, as implied by Eq. (5), the cancellation time scales with the square of the distance. The maximum distance in an axis is $L / 2$ so in the full space must be $(N d)^{1 / 2} L / 2$. This, combined with $L=N^{1 / d}$, explains the behavior that we observe.

This conclusion- that the cancellation time grows faster than the difference of energy between the fermionic and the bosonic ground states- is a consequence of choosing initial configurations at random. In fact, in FMC one has the freedom to choose other relationships between plus and minus walkers. One important option is to start with a pair of walkers with all the coordinates of the particles equal except for one pair of particles that have their coordinates interchanged- that is that 
undergo a pair permutation. Interchanging the nearest pair of like-spin particles gives the smallest initial Euclidean separation of the walkers.

In our experiments, we have studied two alternative strategies for generating initial pairs of walkers. The coordinates of the plus walker are chosen at random in the periodic box. We set the coordinates of the minus walker to be the same except for two particles whose coordinates are interchanged. In the first case, we choose this pair at random, and in the second, we exchange the closest pair. Following our previous explanation, we can see that in the first case, the cancellation time must scale as

$$
t_{c}=C N^{2 / d}
$$

and this is what we get as shown in Figure 2. The parameters obtained from fits to Eq. (7) are $C=0.165,0.25$ for $d=2,3$, respectively.

The results obtained for the second case, which is the one used by FMC, are shown in Figure 3. In this case the cancellation time is independent of the number of particles. This indicates that, at least for the free system, the cancellation of pairs will remain an efficient tool when the number of particles of the system becomes bigger.

It is qualitatively plausible that the mean time to cancellation is smaller when pairs are created by permuting the closest pair; the average separation of close pairs is smaller than that of pairs chosen at random. This is illustrated in Figures 4 and 5, where the average pair separation in the free system is compared when pairs are chosen at random versus as closest, in the cases of $d=2,3$. This was in fact the motivation for using close pairs in the formulation of Fermion Monte Carlo.

There is another aspect that is present in FMC that we have not taken into account. This is the fact that in FMC the pairs may produce new pairs- the process we call "repairing" - while in our model the pairs can only annihilate. We simulate repairing by artificially creating a new pair of walkers in the present position of the pair with probability $p \delta \tau$, with $p$ a constant and $\delta \tau$ the time step. Of course, now the cancellation time refers to the total time needed to annihilate the initial pair and all its offspring. An immediate question is whether the inclusion of branching modifies the scaling of the cancellation time with the number of particles. We studied this and concluded that the cancellation time becomes longer but the tendency remains the same.

\section{Analytical results for the free gas}

When a pair moves according to correlated sampling for the free gas, the Euclidean distance between the walkers diffuses in one dimension. Because we use periodic boundary conditions, the walker is absorbed at $x=0$ and $x=Z$. The orientation of the pair is not necessarily parallel to any coordinate axis so that $Z$ varies from $L$ to $d^{1 / 2} L$ where $L$ is the length of a side of the box.

The evolution simply requires the solution of the equation

$$
\frac{D}{2} \frac{\partial^{2} G\left(x, x_{0} ; t\right)}{\partial x^{2}}-\frac{\partial G\left(x, x_{0} ; t\right)}{\partial t}=0
$$

where $D$ is the diffusion constant and with the initial condition $G\left(x, x_{0} ; 0\right)=$ 
$\delta\left(x-x_{0}\right)$. We can write

$$
G\left(x, x_{0} ; t\right)=\sum_{k=1}^{\infty} \phi_{k}(x) \phi_{k}\left(x_{0}\right) e^{-E_{k} t}
$$

where

$$
-\frac{D}{2} \frac{\partial^{2} \phi_{k}(x)}{\partial x^{2}}=E_{k} \phi_{k}(x)
$$

with $\phi_{k}(0)=\phi_{k}(Z)=0$. This leads to the solutions

$$
\phi_{k}(x)=\sqrt{\frac{2}{Z}} \sin \left(\frac{k \pi x}{Z}\right) \quad E_{k}=\frac{D(k \pi)^{2}}{2 Z^{2}} .
$$

In order to calculate the cancellation time of a pair, we build

$$
\rho\left(x_{0} ; t\right)=\int_{0}^{Z} G\left(x, x_{0} ; t\right) d x
$$

the probability of survival of a pair initially at a distance $x_{0}$ during a time $t$ $\left(\rho\left(x_{0} ; 0\right)=1\right.$ and $\left.\rho\left(x_{0} ; \infty\right)=0\right)$. The probability of cancellation is $1-\rho\left(x_{0} ; t\right)$. If we differentiate this with respect to time, we obtain the distribution of times of cancellation, and its mean will correspond to the time needed to cancel a pair initially at $x_{0}$. This is

$$
t_{c}\left(x_{0}\right)=\int_{0}^{\infty}\left[-\rho^{\prime}\left(x_{0} ; t\right)\right] t d t
$$

So if we define

$$
c_{k}=\int_{0}^{Z} \phi_{k}(x) d x=\frac{\sqrt{2 Z}}{k \pi}\left[1-(-1)^{k}\right],
$$

we can write

$$
\begin{aligned}
\rho\left(x_{0} ; t\right) & =\sum_{k=1}^{\infty} c_{k} \phi_{k}\left(x_{0}\right) e^{-E_{k} t} \\
-\rho^{\prime}\left(x_{0} ; t\right) & =\sum_{k=1}^{\infty} c_{k} E_{k} \phi_{k}\left(x_{0}\right) e^{-E_{k} t} \\
t_{c}\left(x_{0}\right) & =\sum_{k=1}^{\infty} \frac{c_{k}}{E_{k}} \phi_{k}\left(x_{0}\right)
\end{aligned}
$$

Consider the function $f(x)=x(Z-x) / D$ and expand it in eigenfunctions $\left\{\phi_{k}(x)\right\}_{k=1}^{\infty}$. The result is

$$
\begin{aligned}
f(x) & =\sum_{k=1}^{\infty} \frac{c_{k}}{E_{k}} \phi_{k}(x)=t_{c}(x) ; \\
t_{c}(x) & =x(Z-x) / D .
\end{aligned}
$$


The spatial variable we are using here is the difference between two variables with perfect negative correlation so $D$ is four times the diffusion constant of one of these variables.

We present the results in Figure 6 and compare them with the function

$$
t_{c}=A r(B-r)
$$

where for a three-dimension system and branching term of $p=1.15$, we obtain $A=0.79,0.82$ and $B=3.07,5.96$ for $N=10,80$, respectively. The fits are very good.

\section{Harmonic oscillator}

We use the harmonic oscillator as a simple model of an interacting system. The diffusion is described by the exact Green's function [4], modified by the symmetric ground state [5]. This is

$$
\frac{e^{N d \tau / 2} \phi(X) G(X, Y ; \tau)}{\phi(Y)}=\frac{1}{\left(2 \pi e^{-\tau} \sinh \tau\right)^{N d / 2}} \exp \left[\frac{\left(X-e^{-\tau} Y\right)^{2}}{2 e^{-\tau} \sinh \tau}\right]
$$

where $\phi(X)=\exp \left(-X^{2} / 2\right)$. In the case of the harmonic oscillator, the box is not necessary and we have sampled the initial position of the walkers according to $|\phi(X)|^{2}$.

The situation is now different from that in the free gas case since the size of the system does not grow to keep the density constant. Moreover, the attraction provided by the harmonic oscillator potential causes the walkers to stay close to each other. Both facts will be reflected in the behavior of the cancellation time with the number of particles. We will study the same three situations considered in the case of the free gas.

Let us begin with the case where the two walkers in the pair are sampled independently from the ground-state wave function. Figure 7 shows that the cancellation time behaves as

$$
t_{c}=A+B \ln (N)
$$

with $A=0.67,0.83$ and $B=0.49,0.50$ for $d=2,3$, respectively. The cancellation time grows with the number of particles but slower than any positive power of $N$. This behavior is better than the two first cases for the free gas. We can also see that, contrary to the behavior of the free gas, the cancellation time is larger for the three dimensional case than for the two-dimensional case.

This first case is the worst of the three cases, since all the particles in both walkers are chosen independently. In the rest of the study, all the particles in the plus walker are chosen independently but all the particles of the minus walker but two are chosen to have the same coordinates as the corresponding particle in the plus walker. This pair of particles, whose coordinates are interchanged to create the minus walker from the plus walker, can be chosen at random or chosen as the nearest pair of particles in the plus walker.

Figure 8 shows that, when the pair of particles to be exchanged is randomly chosen, the cancellation time is independent of the number of particles.

Finally, if the exchanged pair is the closest pair, the cancellation time actually 


\section{Harmonium atom}

Finally we consider a modification of the harmonic oscillator by including a repulsive potential quadratic in the distance between pairs of particles. We use the Hamiltonian

$$
H=-\frac{\hbar^{2}}{2 m} \sum_{k=1}^{N} \nabla_{x_{k}}^{2}+\frac{1}{2} m N \omega^{2} \sum_{k=1}^{N} x_{k}^{2}-\frac{1}{2} m \omega^{\prime 2} \sum_{l>k}^{N} x_{k l}^{2}
$$

where $\boldsymbol{x}_{k}$ is a $d$-dimensional vector and $x_{k l}$ is the distance between particle $k$ and particle $l$. We have multiplied the attractive part by $N$ to compensate for the smaller number of particles as compared to the number of pairs. Making the change of variables $\boldsymbol{r}_{k}=\sqrt{\frac{m \sqrt{N} \omega}{\hbar}} \boldsymbol{x}_{k}$ and defining $\beta=\frac{\omega^{\prime}}{\omega}$, we can write

$$
H=\frac{\hbar \sqrt{N} \omega}{2}\left[-\sum_{k=1}^{N} \nabla_{r_{k}}^{2}+\sum_{k=1}^{N} r_{k}^{2}-\frac{\beta^{2}}{N} \sum_{l>k}^{N} r_{k l}^{2}\right]
$$

Since the potential is a quadratic form, we can look for a further change of variables which diagonalizes it. This is provided by

$$
\begin{aligned}
\boldsymbol{R}_{1} & =\frac{1}{\sqrt{N}} \sum_{k=1}^{N} \boldsymbol{r}_{k} \\
\boldsymbol{R}_{k} & =\frac{1}{\sqrt{k(k-1)}}\left(\sum_{l=1}^{k-1} \boldsymbol{r}_{l}-(k-1) \boldsymbol{r}_{k}\right) \quad k=2, \ldots, \mathrm{N} .
\end{aligned}
$$


In terms of these new variables, we can rewrite the Hamiltonian as

$$
H=\frac{\hbar \sqrt{N} \omega}{2}\left[-\sum_{k=1}^{N} \nabla_{R_{k}}^{2}+R_{1}^{2}+\left(1-\beta^{2}\right) \sum_{k=2}^{N} R_{k}^{2}\right]
$$

obtaining a system of uncoupled harmonic oscillators if $\gamma^{2}=1-\beta^{2} \geq 0$.

We have studied this using $d=3$ and $\gamma=0.1$ and, as in the previous sections, emphasizing the behavior with $N$ and with three choices for creating pairs. The results are presented in Figures 11, 12 and 13. The first one corresponds to the case where the walkers in the pair are chosen independently. The cancellation time increases linearly with the logarithm of $N$ as was true in the case without repulsion $(\gamma=1)$. We fit using Eq.(21) and obtained $A=8.1$ and $B=5$.

Figure 12 shows the results when the second walker is formed by exchanging a randomly chosen pair of particles in the first walker. We obtain the same behavior as in the case $\gamma=1$ : a constant cancellation time as a function of the number of particles.

Finally, in Figure 13 the pair of particles exchanged is the closest. Here the cancellation time decreases when the number of particles increases. We used Eq.(22) to fit and obtained $C=19.8$

In all cases the cancellation time is greater than when there is no no repulsion $(\gamma=1)$. A comparison between the new values of $A, B, C$ and the constant cancellation time for the second case compared to those in the previous section (which correspond to no repulsion) show that they are roughly multiplied by a factor of 10. This can be easily explained by examining the exact Green's function, modified by the symmetric ground state when the oscillator constant is $\gamma$ instead of 1 . This is

$$
\frac{e^{\gamma d \tau / 2} \phi(X) G(X, Y ; \tau)}{\phi(Y)}=\left(\frac{\gamma}{2 \pi e^{-\gamma \tau} \sinh (\gamma \tau)}\right)^{d / 2} \exp \left[-\frac{\gamma\left(X-e^{-\gamma \tau} Y\right)^{2}}{2 e^{-\gamma \tau} \sinh (\gamma \tau)}\right]
$$

where $\phi(X)=\exp \left(-\gamma X^{2} / 2\right)$. It reduces to the case where $\gamma=1$ using $\tau^{\prime}=\gamma \tau$ and $X^{\prime}=\sqrt{\gamma} X$. This shows that the modified cancellation time is just the cancellation time with no repulsion divided by $\gamma$.

\section{A model system}

As a final application, we study how the cancellation time scales with the number of particles in a system whose Green's function is not known exactly. We use an approximate Green function in the short time limit [6]. The latter can be written as:

$$
G(X, Y ; \tau)=\frac{1}{(2 \pi \tau)^{N d / 2}} \exp \left[-\frac{(X-Y-\tau F(Y))^{2}}{2 \tau}\right] \exp \left[\left(E_{T}-E_{L}(X)\right) \tau\right]
$$

We have included importance sampling with a Jastrow approximation to a trial function for the symmetric ground state $\phi(X)$ that determines

$$
F(Y)=\frac{\nabla \phi(Y)}{\phi(Y)}
$$


and the local energy

$$
E_{L}(X)=\frac{H(X) \phi(X)}{\phi(X)}=-\frac{\nabla^{2} \phi(X)}{2 \phi(X)}+V(X)
$$

This short-time Green function implies "drift" and "diffusion" derived from the first (shifted) exponential and a branching term, derived from the second exponential. The Gaussian part has already been used in this paper and we treat it in the same way as for the free and the harmonic systems. However, the branching part implies population variations and causes plus and minus walkers to have different weights given by

$$
w^{ \pm}=\exp \left[\left(E_{T}-E_{L}\left(X^{ \pm}\right)\right) \tau\right]
$$

In order to retain the structure of a population of pairs of walkers, we define

$$
\begin{aligned}
& w_{p}=\min \left(w^{+}, w^{-}\right) \\
& w_{r}=\frac{\max \left(w^{+}, w^{-}\right)-\min \left(w^{+}, w^{-}\right)}{2} .
\end{aligned}
$$

$w_{p}$ gives the expected number of pairs of new walkers generated by the smaller of $w^{+}, w^{-}$; the extra weight $w_{r}$ gives the expected number of additional pairs created by "repairing". This consists in taking the walker with the larger weight, $\max \left(w^{+}, w^{-}\right)$and generating a new partner by exchanging only the closest pair of particles of the original configuration. Because both of the walkers of this new pair have the same expectation for future contributions weighted with antisymmetric test functions, this accounts for all weights and is unbiased.

In studying the time to cancellation, it is not necessary to carry out the branching. Instead we may define:

$$
p_{p}=\frac{w_{p}}{w_{p}+w_{r}}, \quad p_{r}=\frac{w_{r}}{w_{p}+w_{r}}
$$

Then with probability $p_{p}$, the pair continues drift and correlated diffusion, and otherwise the pair "repairs" using the nearest pair permutation as discussed above.

This avoids the necessity for population control and eigenvalue estimation, while correctly balancing the correlated diffusion against its interruption by repairing. It also correctly treats the decreased probability of repairing as the walkers become close and the two weights, $w^{+}$and $w^{-}$, become equal.

We present results with the so-called Pöschl-Teller potential [7]:

$$
V(X)=\sum_{j>i}^{N} V\left(r_{i j}\right)=-\sum_{j>i}^{N} \frac{2 \mu^{2}}{\cosh ^{2}\left(\mu r_{i j}\right)}
$$

We use values of the parameter $\mu$ that give an infinite scattering length [8].

We will use a trial wavefunction of the form

$$
\phi(X)=\prod_{l>k}^{N} f\left(r_{l k}\right)
$$


where the correlation function $f(r)$ is the solution of the equation

$$
-\nabla^{2} f(r)+V(r) f(r)=\lambda f(r) .
$$

We use a healing distance, $d_{h}$ defined by

$$
\frac{\rho}{2} \int_{0}^{d_{h}} f^{2}(r) d \vec{r}=1
$$

and require that $f\left(r \geq d_{h}\right)=1$ and $f^{\prime}\left(d_{h}\right)=0$.

We show in Fig. 14 results for the three dimensional case $(d=3)$ when both walkers of the initial pair are chosen at random inside the box. In this example the efficiency of the cancellation process improves as the number of particles grows. We also show in this figure a fit using Eq. (22) with $C=0.24$. This fit also appeared in the harmonic oscillator case when the plus walker was chosen at random and the minus walker was equal to the plus one except for the permutation of the nearest pair of particles. This holds here without the special choice of initial pairs because repairing is guaranteed to occur sometime in the random walk. The drifting force caused by an attractive potential with a minimum at the origin brings the pairs together, accelerating the annihilation process. Cancellation is as fast as in the case of the harmonic oscillator. In other words, the repairing process, required because an approximate importance function is used in the random walk, accelerates the cancellation process. We expect this to hold quite generally.

\section{Conclusions}

Our study of the dynamics of correlated diffusion has been intended to clarify their use in Quantum Monte Carlo computations of fermionic systems. It is possible, of course, that they may be of wider interest, because in an important sense, they show how correlated random walkers can be made to meet in spaces of very high dimension.

Within the framework of Quantum Monte Carlo, our results confirm that for systems like those we have studied- non-interacting fermions, harmonic oscillator systems, and the extensive gas of atoms interacting by way of the Pöschl-Teller potential- the exponential complexity thought to exist when a fixed node is not imposed can be overcome.

The fact that walkers eventually meet implies that the decay of signal to noise in the limit of very large imaginary time can be overcome with the use of correlated diffusion leading to cancellation. The fact that the time to cancellation scales more slowly than linearly in the number of particles means that a computation will not require exponentially large computing time for large systems.

It remains to be seen whether these conclusions hold more generally, but at face value, they imply strongly that the "fermion sign problem" is not computationally intractable as is widely believed.

\section{Acknowledgments}

We are honored to dedicate this paper to Luciano Reatto in recognition of his decades of contributions to condensed matter physics, for the deep theoretical insights he has derived from Quantum Monte Carlo, and for his friendship. We are grateful to Kevin Schmidt who called our attention to the need to investigate the 


\section{Page 11 of 26

time to cancellation in a systematic way. Joseph Carlson suggested the importance of studying the free-fermion problem as a basic challenge and as a source of insight. This manuscript has benefited greatly from critical comments by David Hardin. F.A.S. acknowledges financial support from the Spanish Ministerio de Educación during his stay at the Lawrence Livermore National Laboratory and from the project FIS2009-07390. F.A.S. and F.P. thank Eric Schwegler and the Quantum Simulation Group for their hospitality during visits to the Livermore Laboratory. This work was performed under the auspices of the Lawrence Livermore National Security, LLC, (LLNS) under Contract No. DE-AC52-07NA27344.

\section{References}

[1]D.M. Arnow, M.H. Kalos, M.A. Lee, and K.E. Schmidt, J. Chem. Phys. 77, 5562 (1982).

[2] M.H. Kalos and F. Pederiva, Phys. Rev. Lett. 85, 3547 (2000).

[3] M.H. Kalos, L. Coletti, and F. Pederiva, J. Low Temp. Phys. 138, 747 (2005).

[4]R.P. Feynman, Statistical Mechanics: A Set of Lectures, Addison-Wesley, Redding, (1992).

[5] M.H. Kalos and K.E. Schmidt, J. Stat. Phys. 89, 425 (1997).

[6] M.H. Kalos and P.A. Whitlock, Monte Carlo Methods, Second Edition, Wiley-Blackwell, (2008).

[7] G. Pöschl and E. Teller, Z. Phys. 83, 143 (1933).

[8]S.Y. Chang, V.R. Pandharipande, J. Carlson and K.E. Schmidt, Phys. Rev. A 70, 043602 (2004). 


\section{FIGURE CAPTIONS}

Figure 1. Behavior of the cancellation time with the number of particles, $N$, moving in a $d$-dimension space. The two walkers in the pair are sampled independently from an uniform distribution. The straight lines show $t_{c} \propto N^{1+2 / d}$ with: dots line for $d=1$, dashes line for $d=2$ and full line for $d=3$.

Figure 2. The same as Figure 1 but the two walkers now have the same coordinates except for two particles,chosen at random, which are exchanged. The straight lines show $t_{c} \propto N^{2 / d}$ using the same convention as in Figure 1.

Figure 3. The same as Figure 2 but the two exchanged particles have been chosen to be the closest among all pairs. The constant lines corresponds to $d=3$ for the full line and $d=2$ for the dashes line.

Figure 4. Average pair separation in a system of $N$ particles when both configurations are chosen at random. The straight line represents $\left\langle r_{i j}>\propto N^{1 / d}\right.$ with: full line for $d=3$ and dashes line for $d=2$.

Figure 5. Average pair separation in a system of $N$ particles when the second configuration is the same as the other except for permuting the closest particle pair. The straight line represents $\left\langle r_{i j}>\propto N^{-1 / d}\right.$ using the same convention as in Figure 4.

Figure 6. The cancellation time, $t_{c}$, as a function of the initial distance of the pair, $r$, for three-dimensional systems with $N=10$ (full line) and $N=80$ (dashes line) particles.

Figure 7. Cancellation time versus the number of particles for the harmonic oscillator when the walkers in the pair are chosen independently. The full line corresponds to $d=3$ and the dashes one to $d=2$.

Figure 8. Cancellation time versus the number of particles for the harmonic oscillator when the pair of particles exchanged in the pair of walkers is chosen at random. The same convention as in Figure 7 is used.

Figure 9. Cancellation time versus the number of particles for the harmonic oscillator when the pair of particles exchanged in the pair of walkers is the closest one. The same convention as in Figure 7 is used.

Figure 10. The cancellation time, $t_{c}$, as a function of the initial distance of the pair, $r$, for a three-dimensional system with $N=640$ particles in a harmonic oscillator potential.

Figure 11. Cancellation time versus the number of particles for Harmonium with $\gamma=0.1$ and $d=3$ when the walkers in the pair are chosen independently.

Figure 12. Cancellation time versus the number of particles for Harmonium with $\gamma=0.1$ and $d=3$ when the pair of particles exchanged in the pair of walkers is chosen at random.

Figure 13. Cancellation time versus the number of particles for Harmonium with $\gamma=0.1$ and $d=3$ when the particles exchanged form the closest pair.

Figure 14. Cancellation time versus the number of particles in the short time limit when the walkers in the pair are chosen independently. 
1

2

3

4

5

6

7

8

9

10

11

12

13

14

15

16

17

18

19

20

21

22

23

24

25

26

27

28

29

30

31

32

33

34

35

36

37

38

39

40

41

42

43

44

45

46

47

48

49

50

51

52

53

54

55

56

57

58

59

60

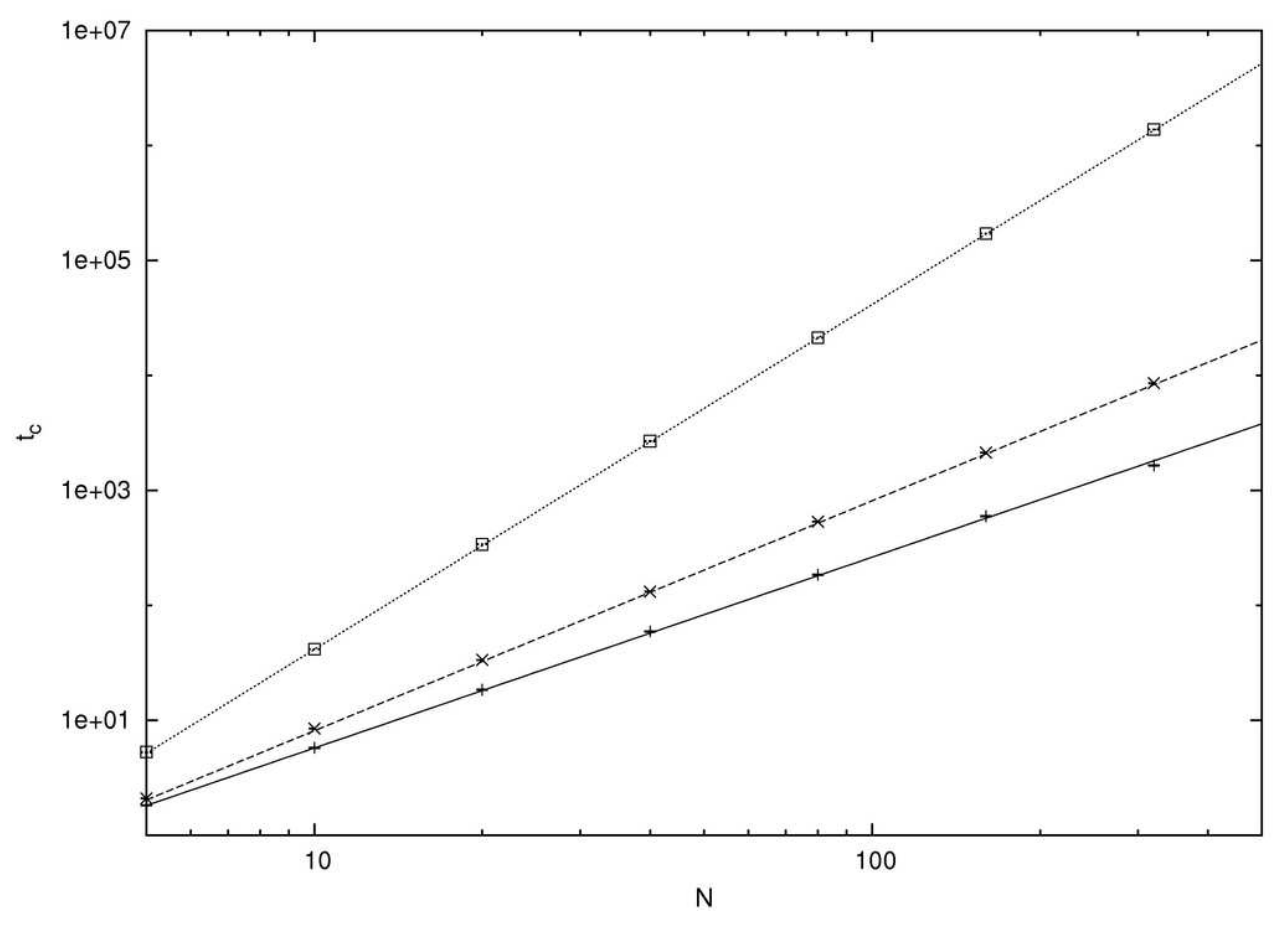

Figure 1

$88 \times 62 \mathrm{~mm}(300 \times 300$ DPI $)$ 


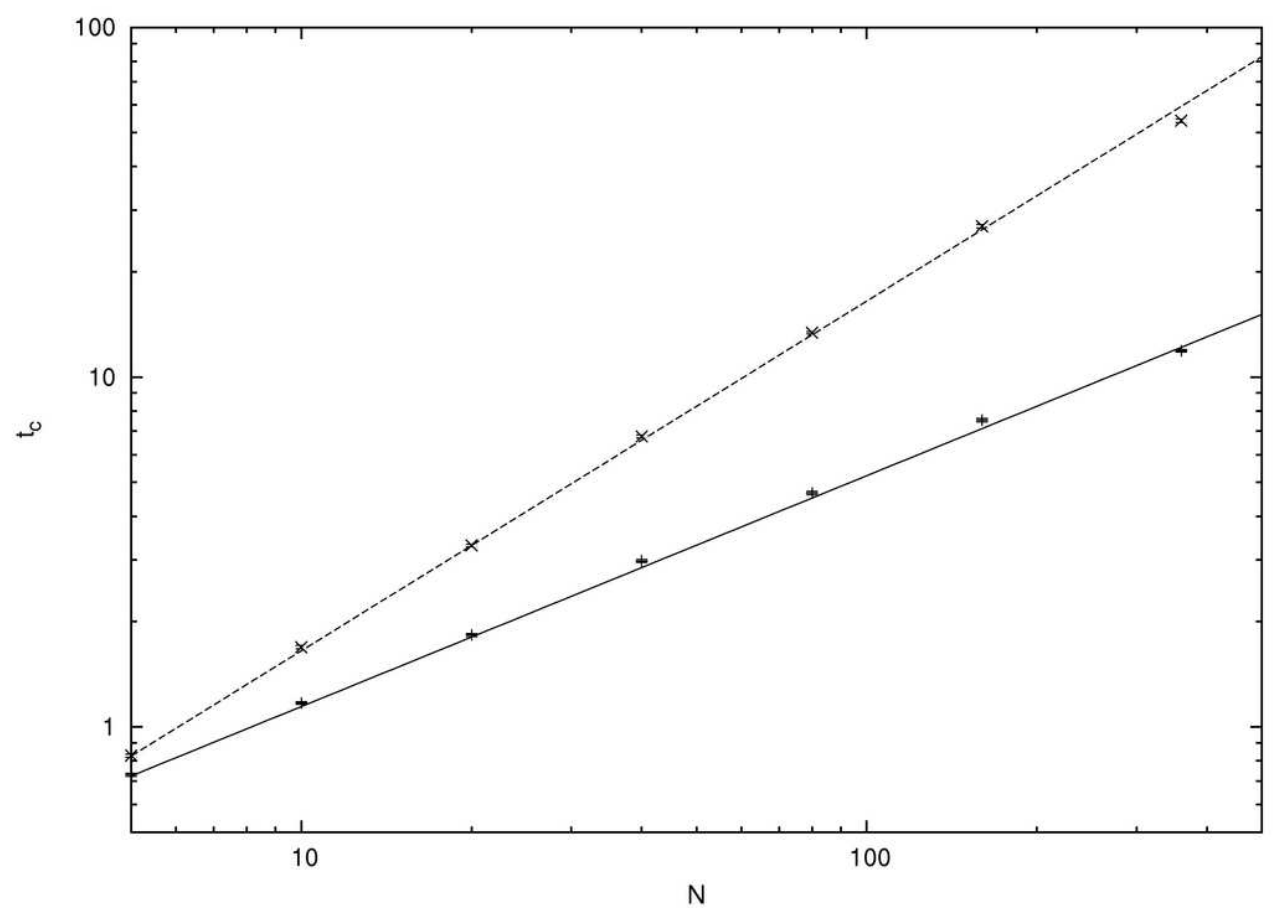

Figure 2

$88 \times 62 \mathrm{~mm}(300 \times 300$ DPI $)$ 


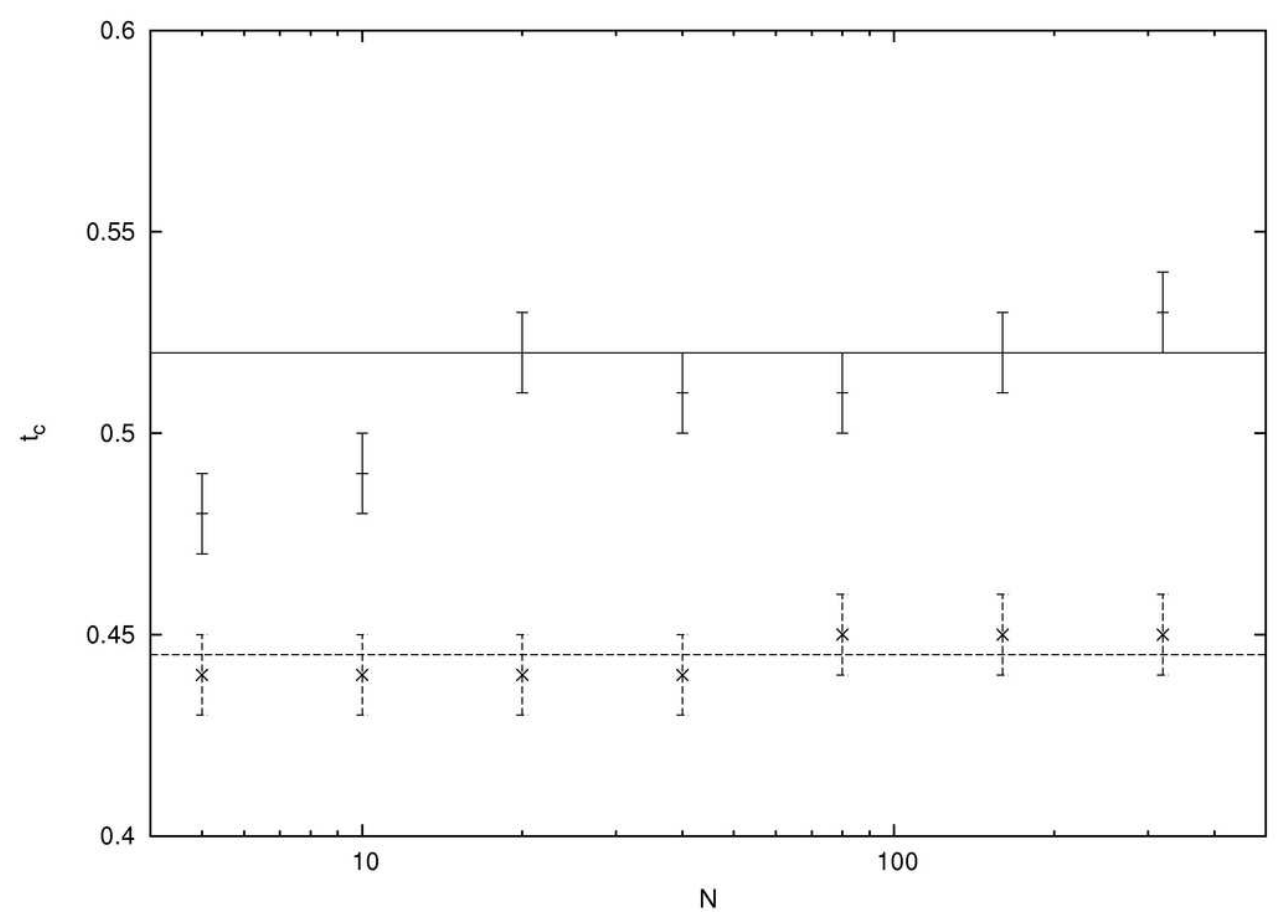

Figure 3

$88 \times 62 \mathrm{~mm}(300 \times 300$ DPI $)$ 


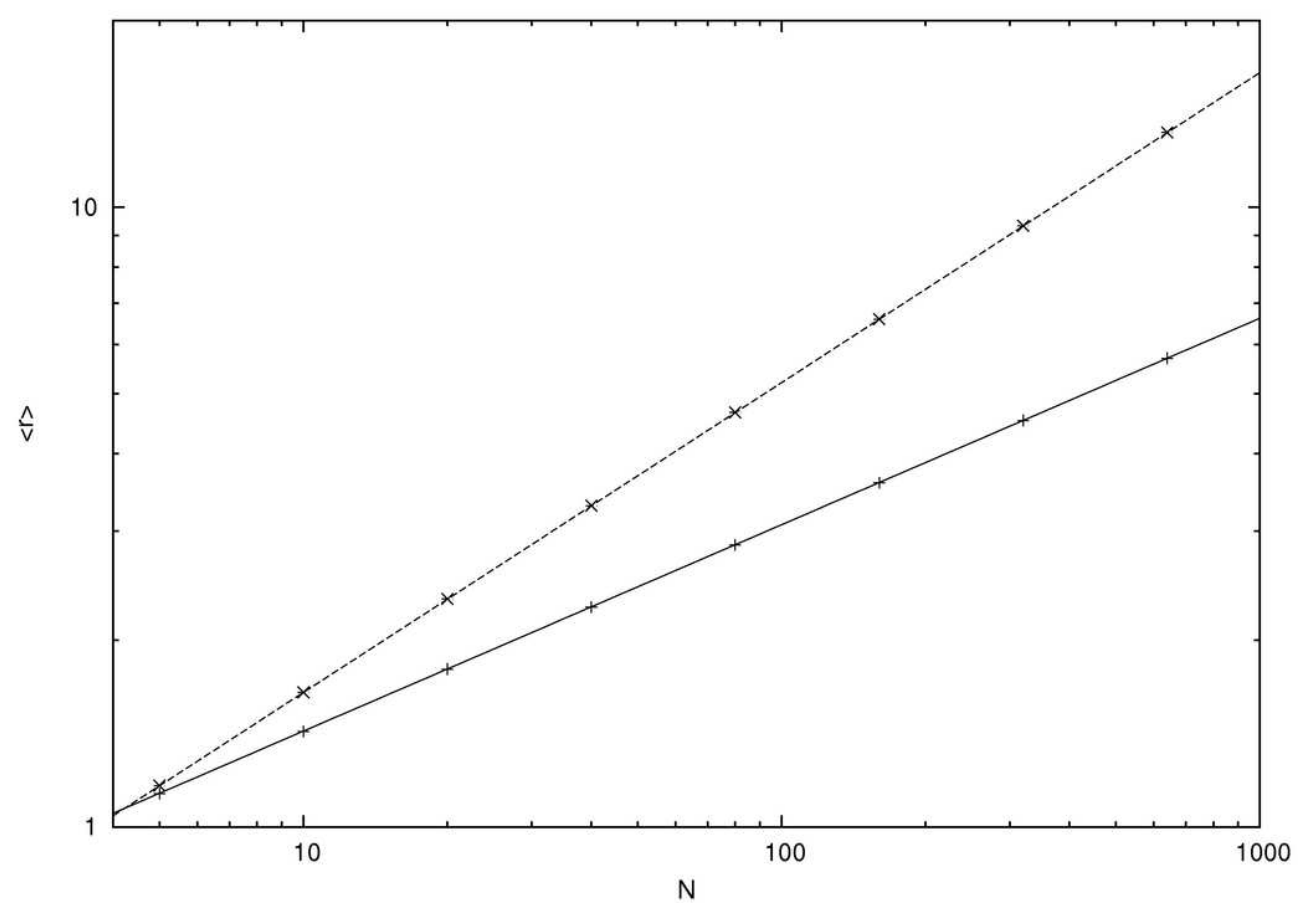

Figure 4 $88 \times 62 \mathrm{~mm}(300 \times 300 \mathrm{DPI})$

URL: http://mc.manuscriptcentral.com/tandf/tmph 
1

2

3

4

5

6

7

8

9

10

11

12

14

15

16

17

18

19

20

21

22

23

24

25

26

27

28

29

30

31

32

33

34

35

36

37

38

39

40

41

42

43

44

45

46

47

48

49

50

51

52

53

54

55

56

57

58

59

60

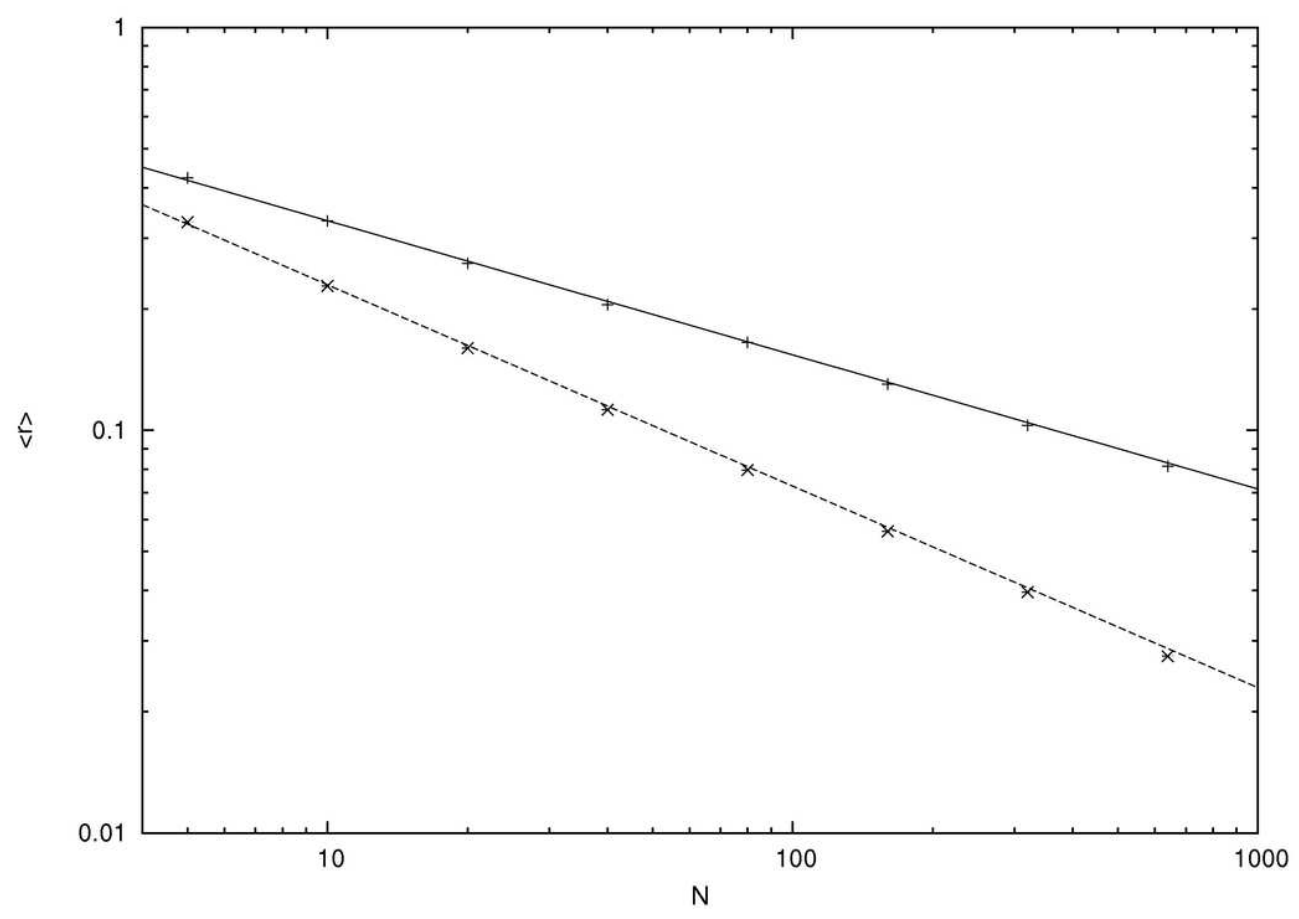

Figure 5

$88 \times 62 \mathrm{~mm}(300 \times 300$ DPI $)$ 


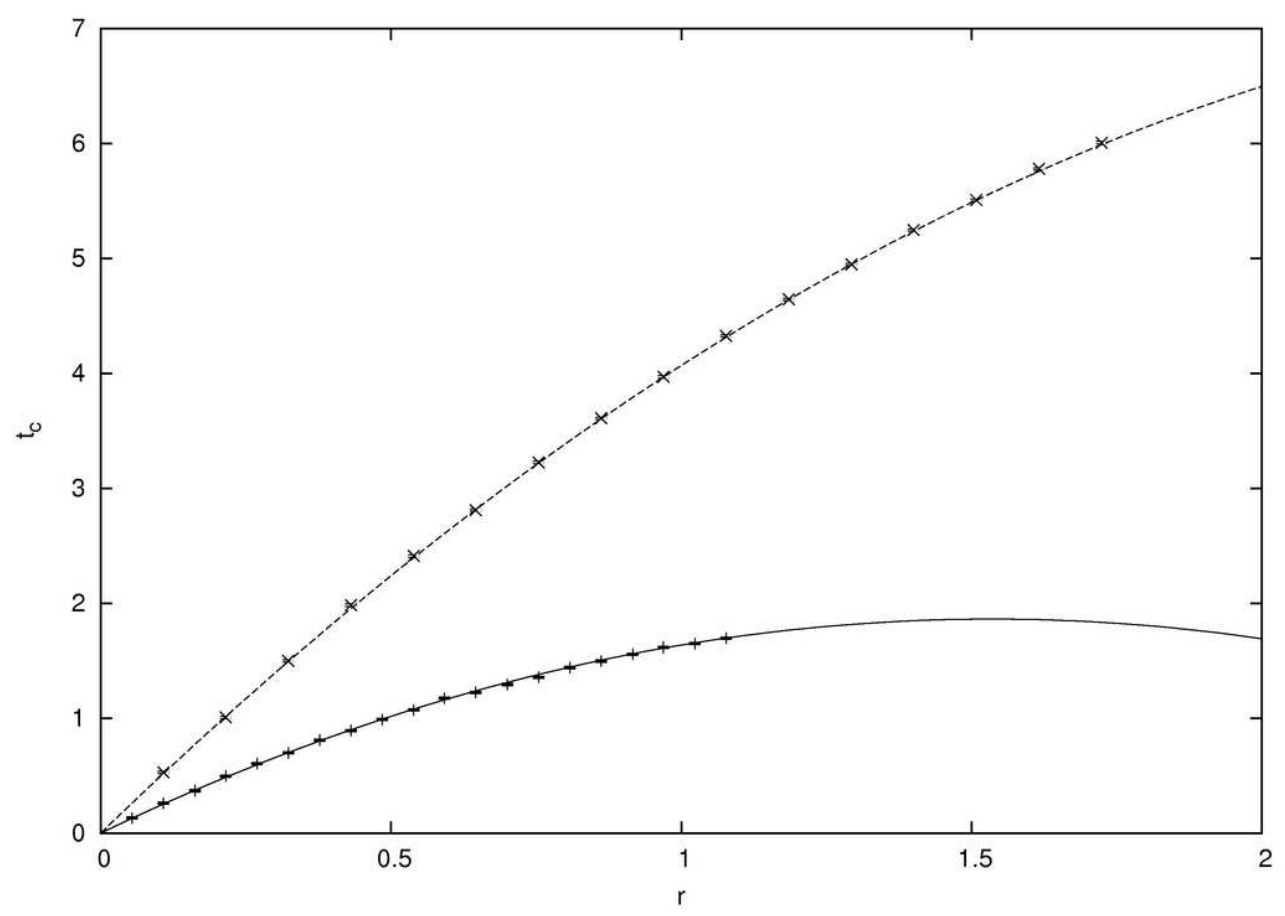

Figure 6

$88 \times 62 \mathrm{~mm}(300 \times 300$ DPI $)$

32

33

34

35

36

37

38

39

40

41

42

43

44

45

46

47

48

49

50

51

52

53

54

55

56

57

58

59

60

URL: http://mc.manuscriptcentral.com/tandf/tmph 


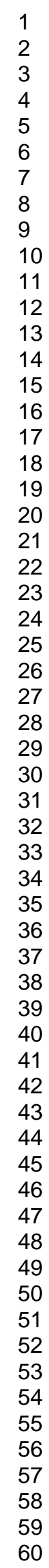

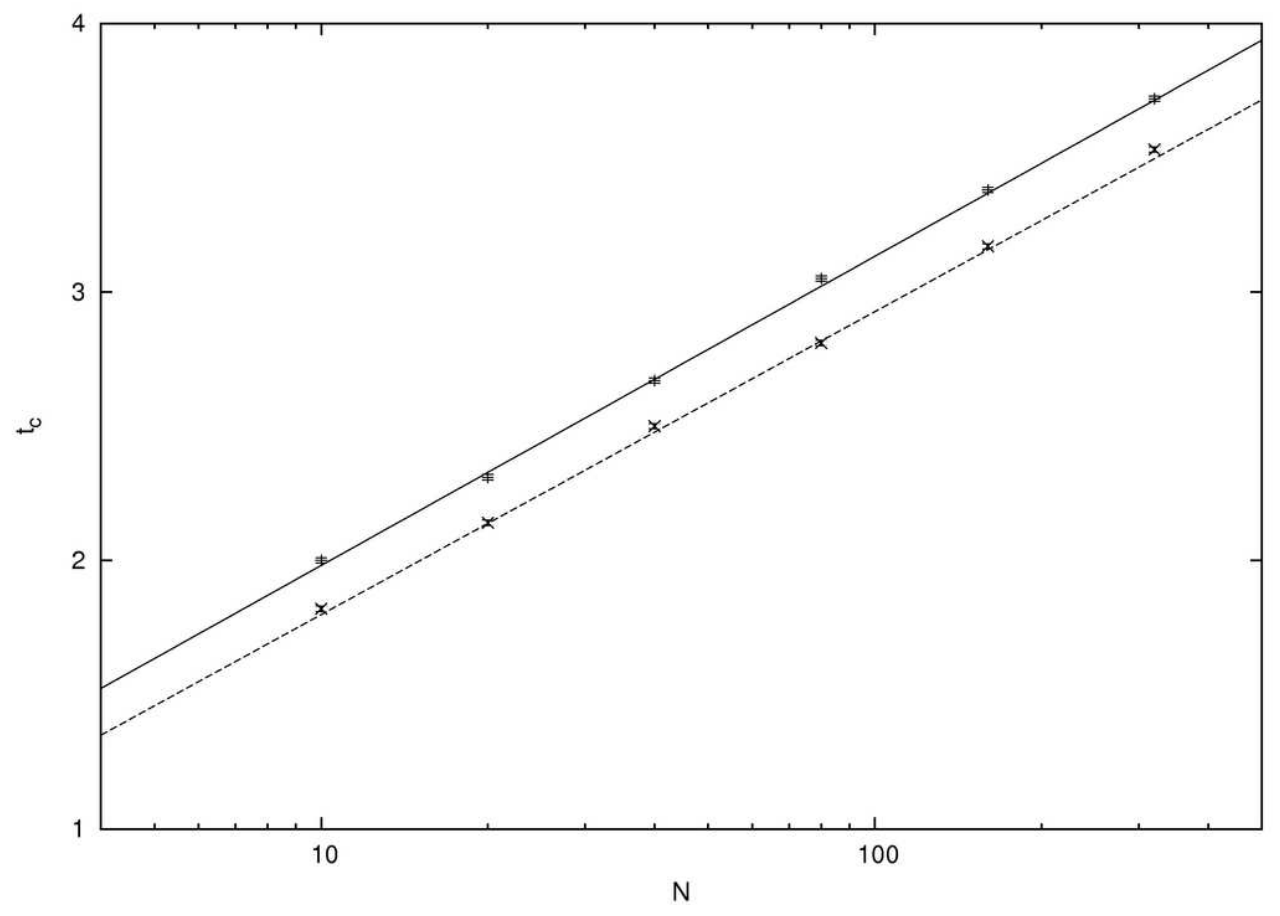

Figure 7

$88 \times 62 \mathrm{~mm}(300 \times 300$ DPI $)$ 


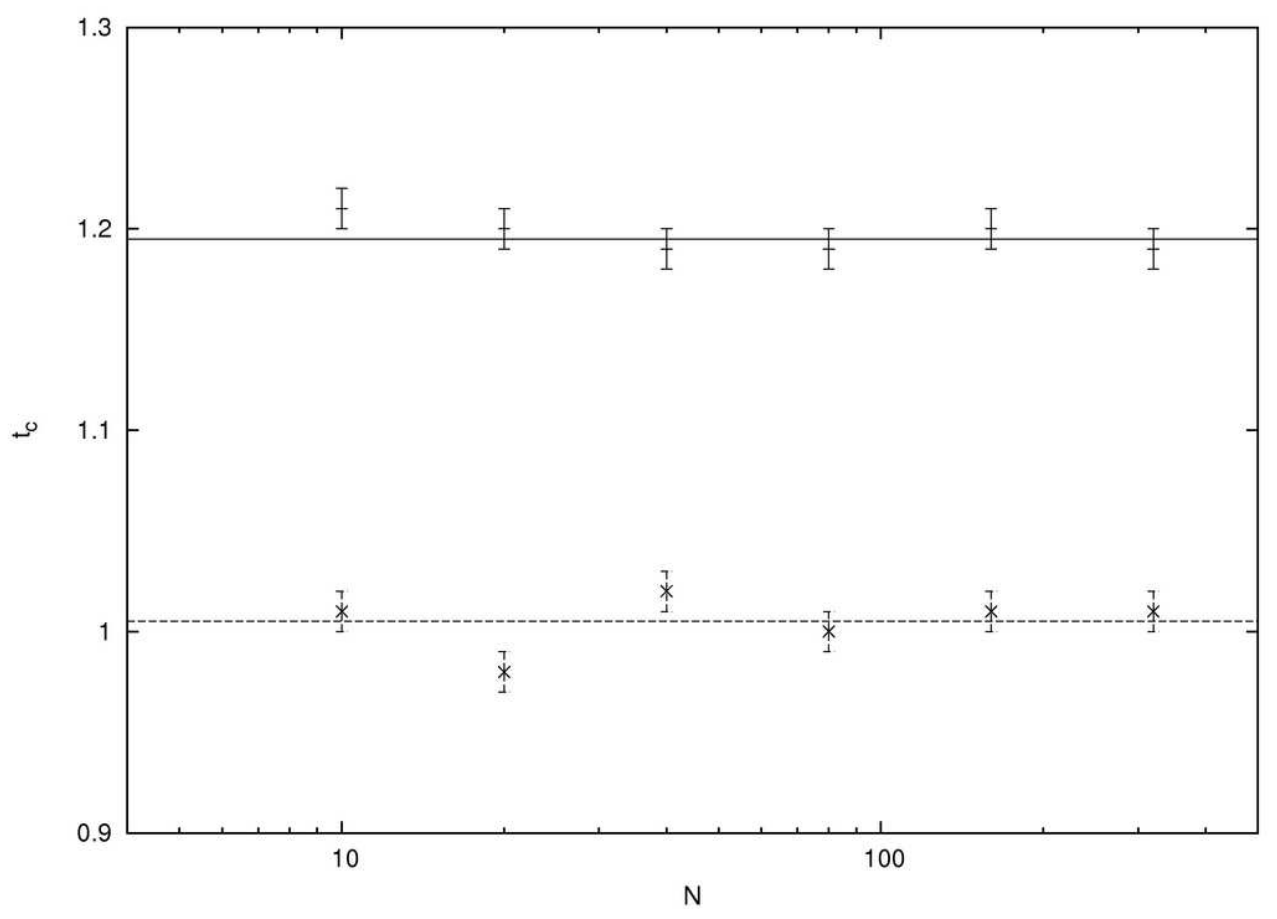

Figure 8

$88 \times 62 \mathrm{~mm}(300 \times 300 \mathrm{DPI})$

URL: http://mc.manuscriptcentral.com/tandf/tmph 
1

2

3

4

5

6

7

8

9

10

11

12

14

15

16

17

18

19

20

21

22

23

24

25

26

27

28

29

30

31

32

33

34

35

36

37

38

39

40

41

42

43

44

45

46

47

48

49

50

51

52

53

54

55

56

57

58

59

60

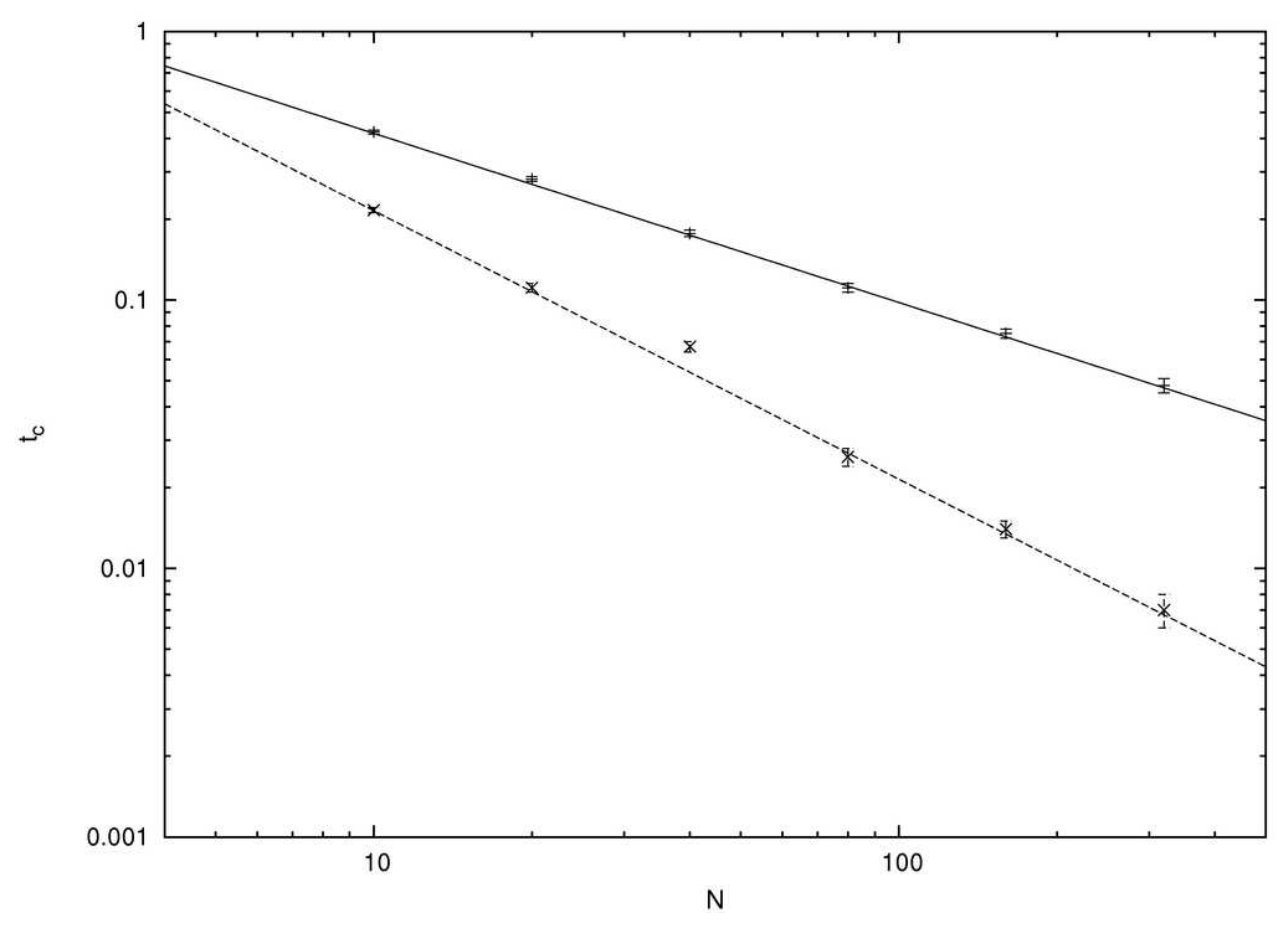

Figure 9

$88 \times 62 \mathrm{~mm}(300 \times 300$ DPI $)$ 


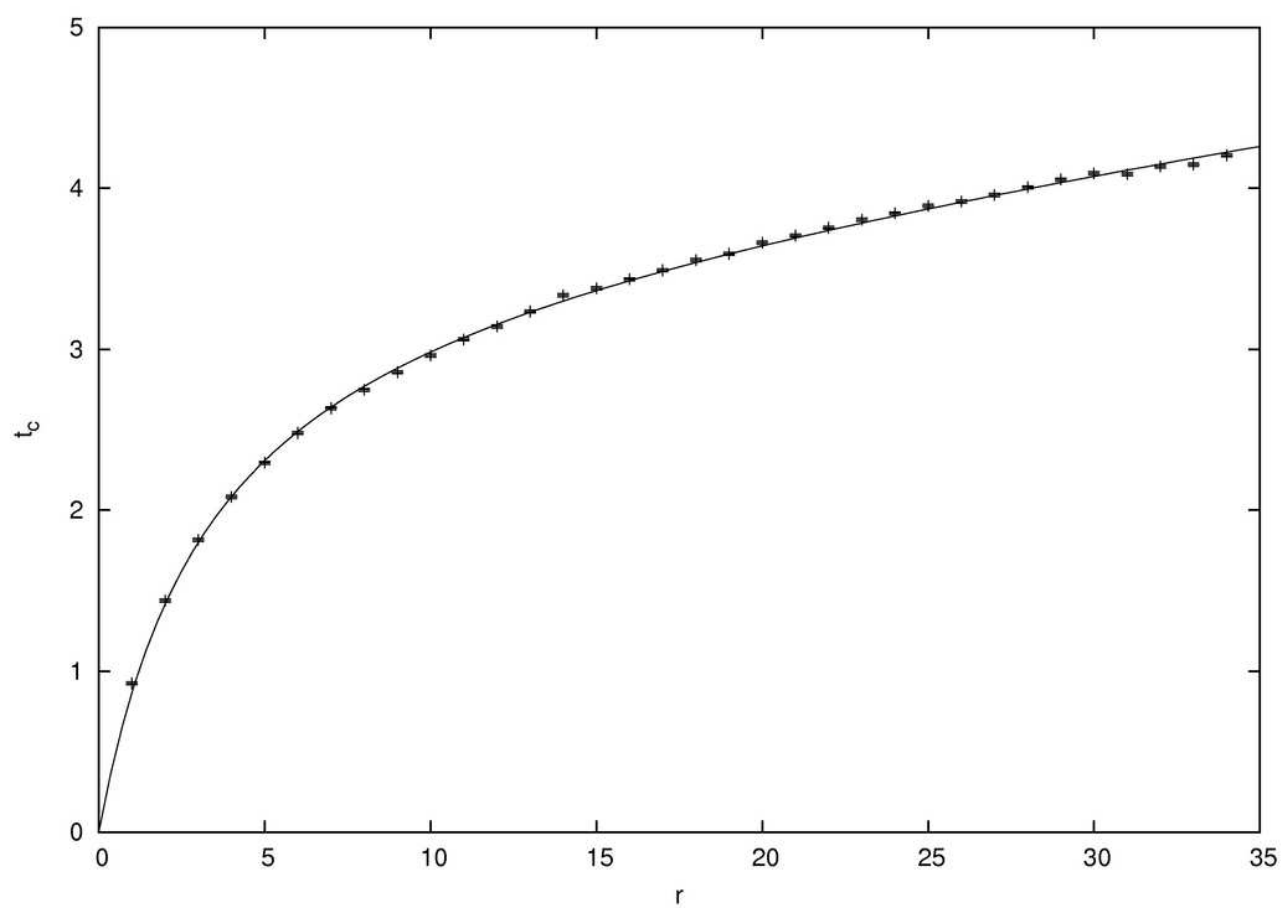

Figure 10

$88 \times 62 \mathrm{~mm}(300 \times 300 \mathrm{DPI})$

URL: http://mc.manuscriptcentral.com/tandf/tmph 


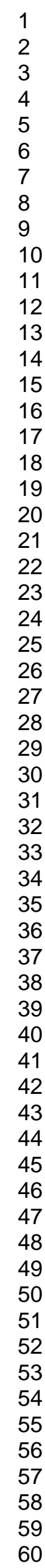

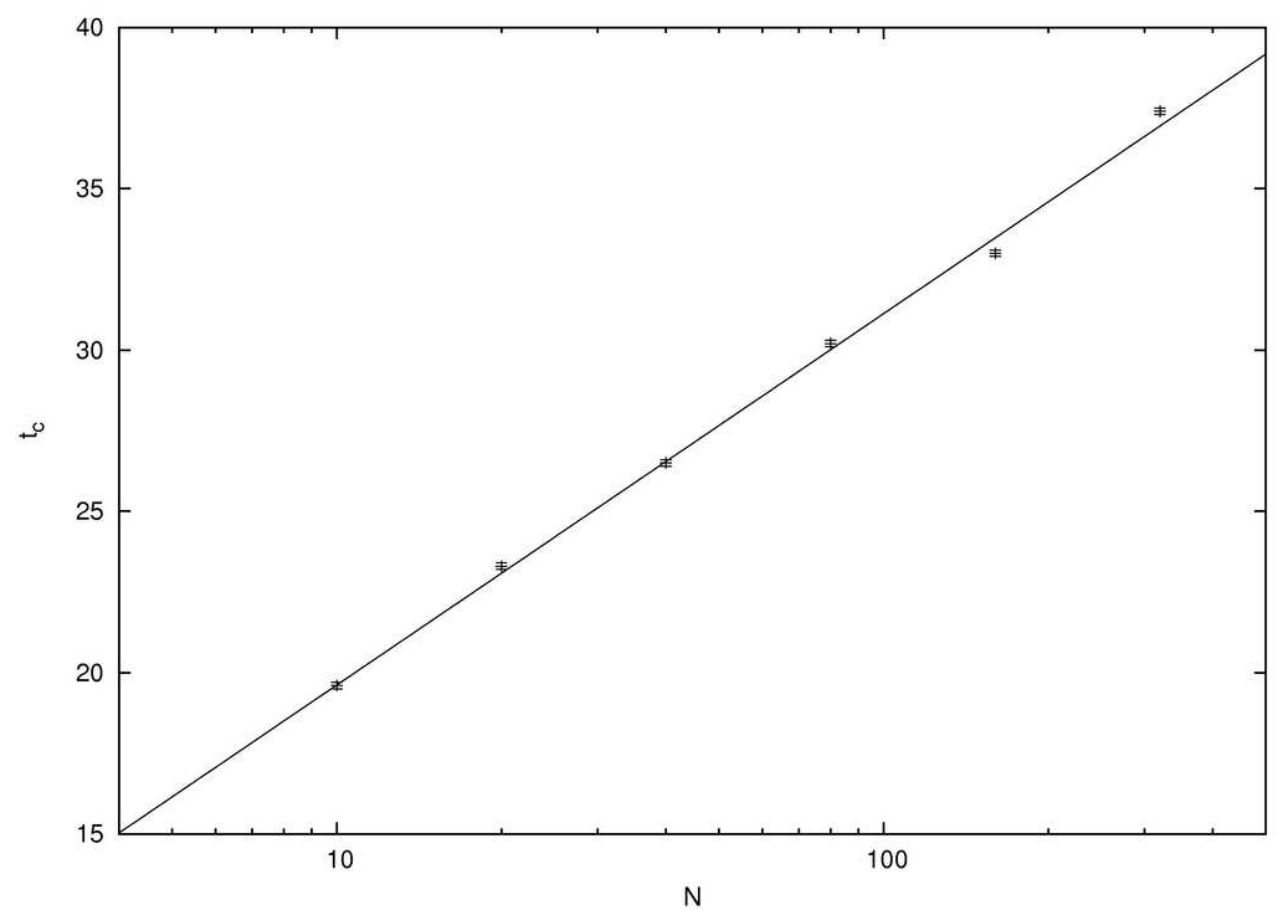

Figure 11

$88 \times 62 \mathrm{~mm}(300 \times 300$ DPI $)$ 


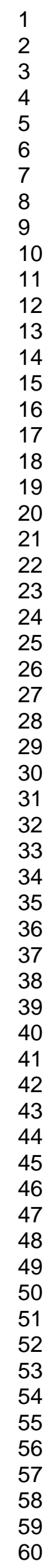

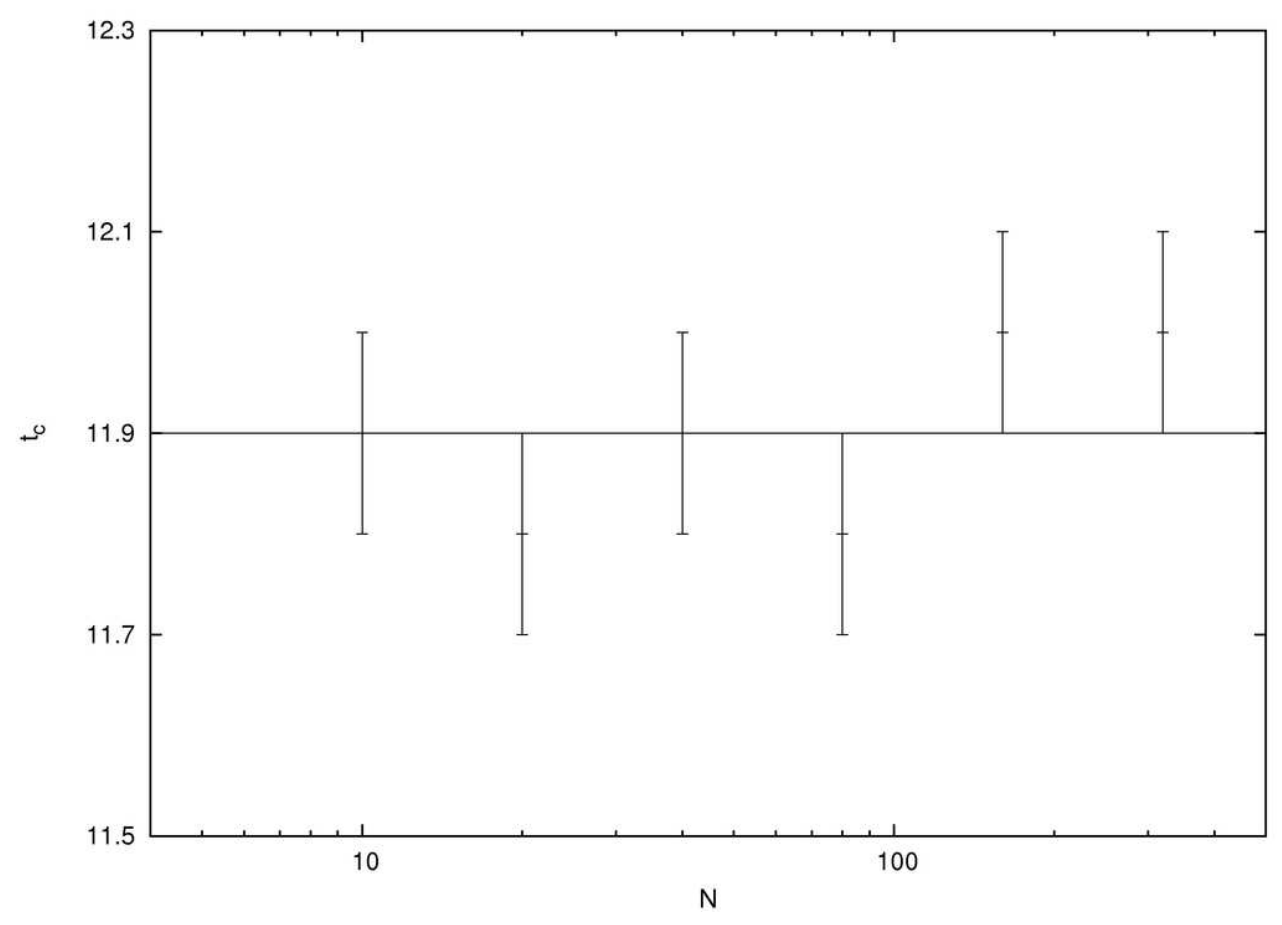

Figure 12

$88 \times 62 \mathrm{~mm}(300 \times 300$ DPI $)$

URL: http://mc.manuscriptcentral.com/tandf/tmph 


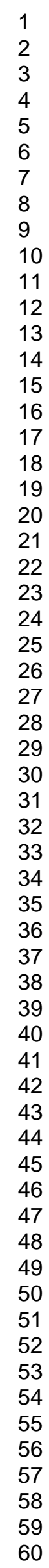

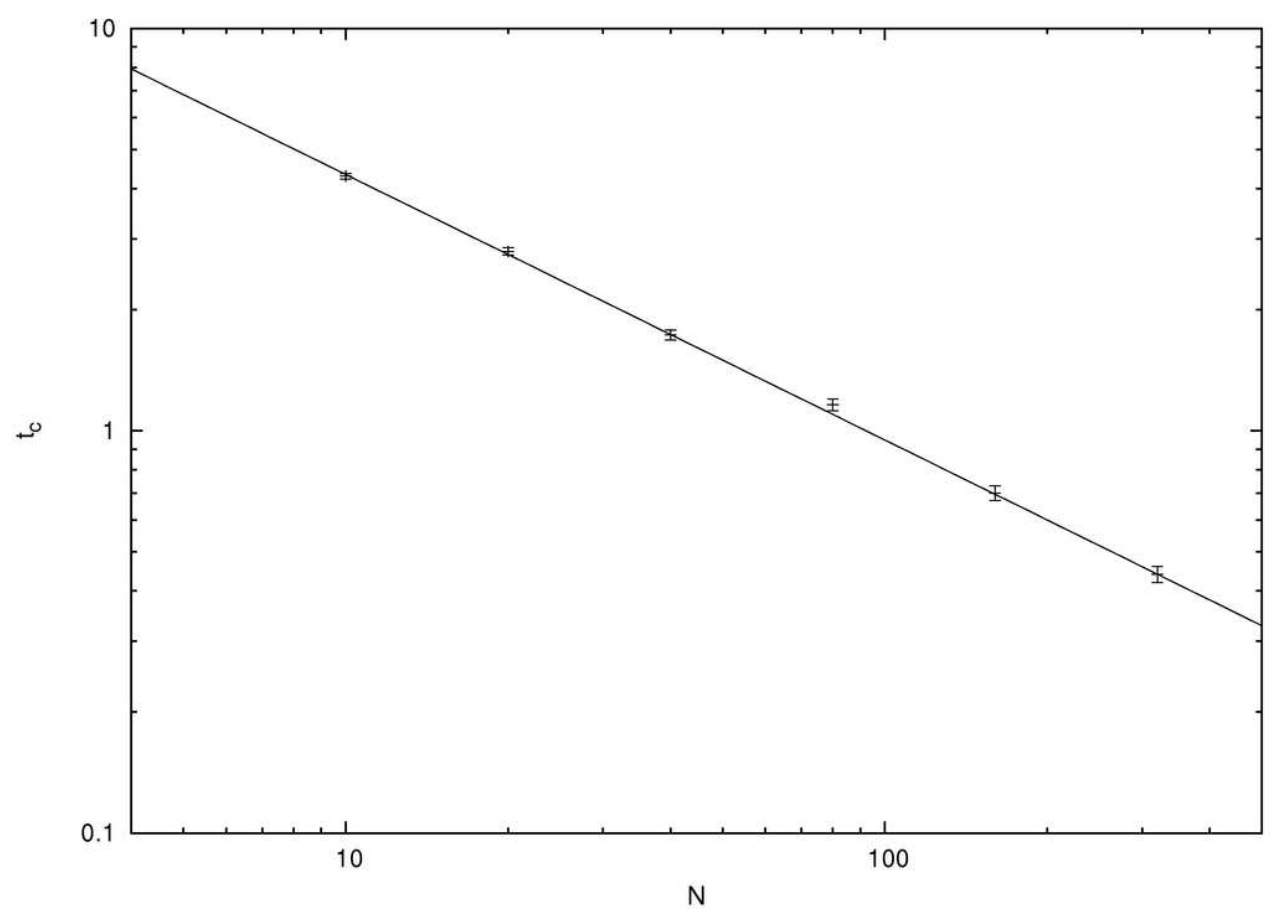

Figure 13

$88 \times 62 \mathrm{~mm}(300 \times 300$ DPI $)$ 


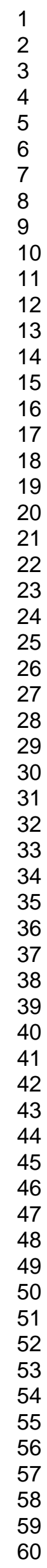

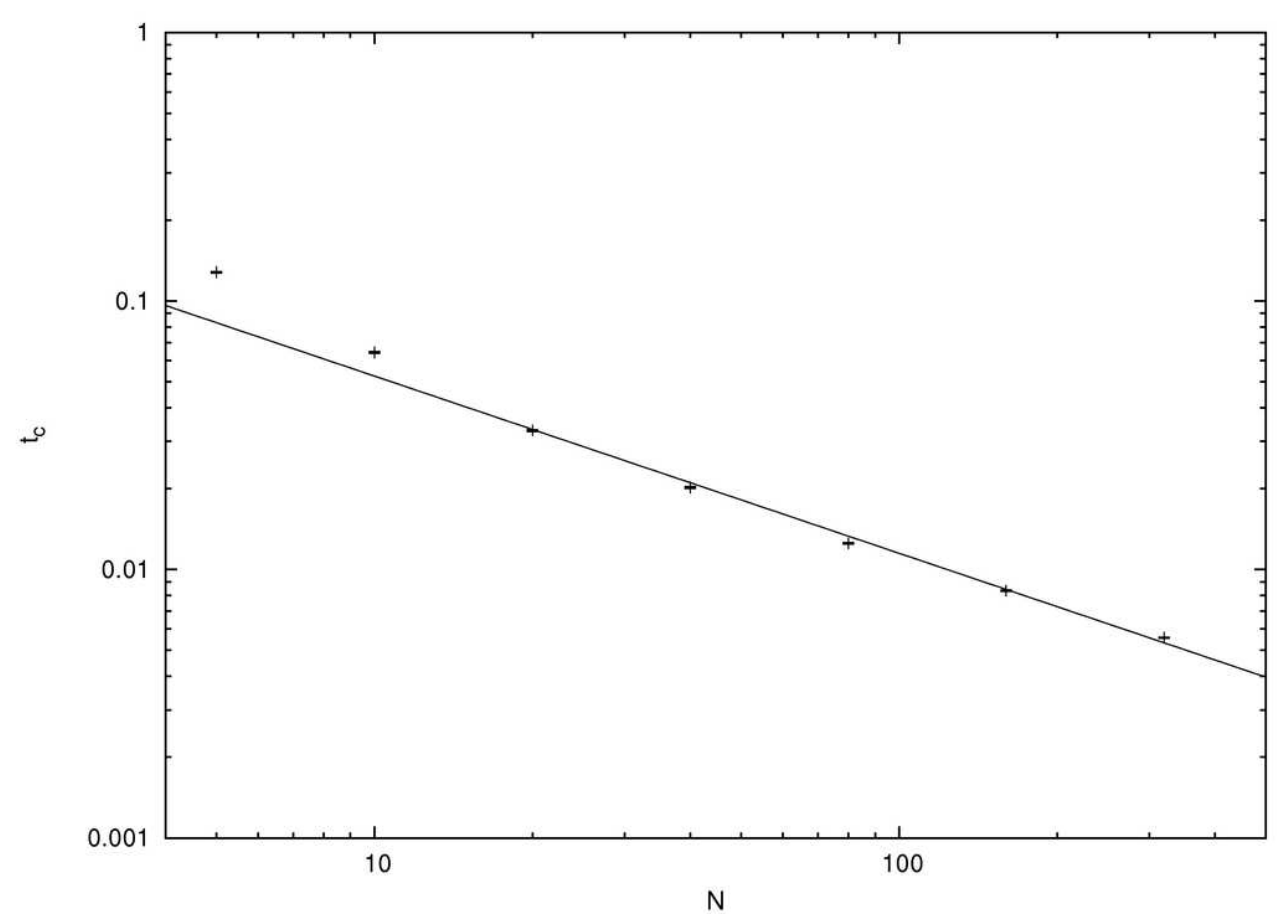

Figure 14

$88 \times 62 \mathrm{~mm}(300 \times 300$ DPI $)$

URL: http://mc.manuscriptcentral.com/tandf/tmph 